\title{
An Insight to Middleware Technologies used for Cloud Computing Frameworks: From the Perspective of Successful Enterprise Application Integration
}

\author{
Osama Harfoushi ${ }^{1} \&$ Ruba Obiedat ${ }^{1}$ \\ ${ }^{1}$ Department of Business Information Technology, The University of Jordan, Amman, Jordan \\ Correspondence: Osama Harfoushi, Department of Business Information Technology, The University of Jordan, \\ Amman, Jordan, Tel: 962-795-707-657. E-mail: o.harfoushi@ju.edu.jo
}

Received: July 27, 2018

Accepted: August 8, 2018

Online Published: August 28, 2018

doi:10.5539/mas.v12n9p234

URL: https://doi.org/10.5539/mas.v12n9p234

\begin{abstract}
At present, the use of middleware technologies has been observed to be used by the companies for successful enterprise application integration (EAI). The EAI can helps in integrating the data and information relating to the systems. Thus, the study focuses on highlighting a brief overview of the EAI along with its architecture. The study has also focused on providing a concise description of all the middleware technologies and the frameworks of cloud computing. In addition, the research study has also focused on using qualitative approach collecting data and information for obtaining data relating to the middleware technologies with respect to the cloud computing and its alignment with EAI. Thus, it can be stated that the use of the middleware technologies can significantly help the companies to cloud computing framework.
\end{abstract}

Keywords: middleware technologies, cloud computing, enterprise application integration (EAI)

\section{Introduction}

Enterprise application integration (EAI) is better understood as a technical term, which has been used since the early 2000s. Conceptually, it is an approach, which helps in offering interoperability amid numerous disparate mechanisms that eventually form a distinctive enterprise infrastructure (MuleSoft Inc., 2018). EAI is also described as an effective strategy of systems integration, which assists in developing data, as well as, process incorporation within a computer-based information system (van Sinderen et.al, 2012). Evidence suggests that the solutions associated with EAI often consider distinct middleware models and approaches with the intention of standardising and centralising incorporation practices throughout a viable enterprise infrastructure (MuleSoft, Inc., 2018). From a technical perspective, middleware and its distinctive technologies are related to EAI in such a way that it is possible to exchange data amid two or more software applications successfully (Nardi et.al, 2011).

Middleware is often viewed as computer software, which is mostly used for the purpose of using along with distributing, as well as, compositing sorts of applications. Some of the middleware technologies include message oriented middleware (MOM), transaction processing (TP) monitors, and object request broker (ORB) among others. These middleware technologies are identified to be used in the frameworks of cloud computing from the standpoint of successful EAI (Kalyani, 2012).

Cloud computing is described as a large-scale distributed paradigm of computing, which is driven by the factors of economies of scale and computer resources, which are virtualised in nature. To be precise, it is defined as a pool of virtualised computer resources based on which dynamically-scalable, services, and storage are offered to the external users as per their demand through internet. The typical facets of cloud computing encompass resource planning, on-demand self-service, rapid elasticity, measured service, and broad network access (Chen, 2010). Considering the present trends and challenges witnessed in EAI, this research paper aimed at developing insights to various middleware technologies that are used for cloud computing frameworks on the behalf of efficient EAI form.

\section{Literature Review}

\subsection{A Brief Overview of EAI's Architectures}

According to van den Bosch et.al. (2010), the requirement for integrating applications is rapidly growing in the 
present day context, as large number of business entities have been observed to be significantly relying on information technology (IT). For instance, technologies such as service-oriented architecture (SOA) takes into concern certain best practices that are developed within the field of EAI domain. These technologies further lead towards leveraging the appearance of EAI in the modern globalised world (van den Bosch et.al, 2010). Based on the observation made by Ritter et.al. (2017), the evolution of new IT trends, as well as, application scenarios certainly posed threats or challenges to EAI. These threats or challenges can be related to the factors concerning Microservices and Cloud computing along with Mobile Computing (Ritter et.al, 2017). The below portrayed diagram (Figure 1) will certainly provide a blueprint to the IT trends since the year 2005 and their association with EAI.

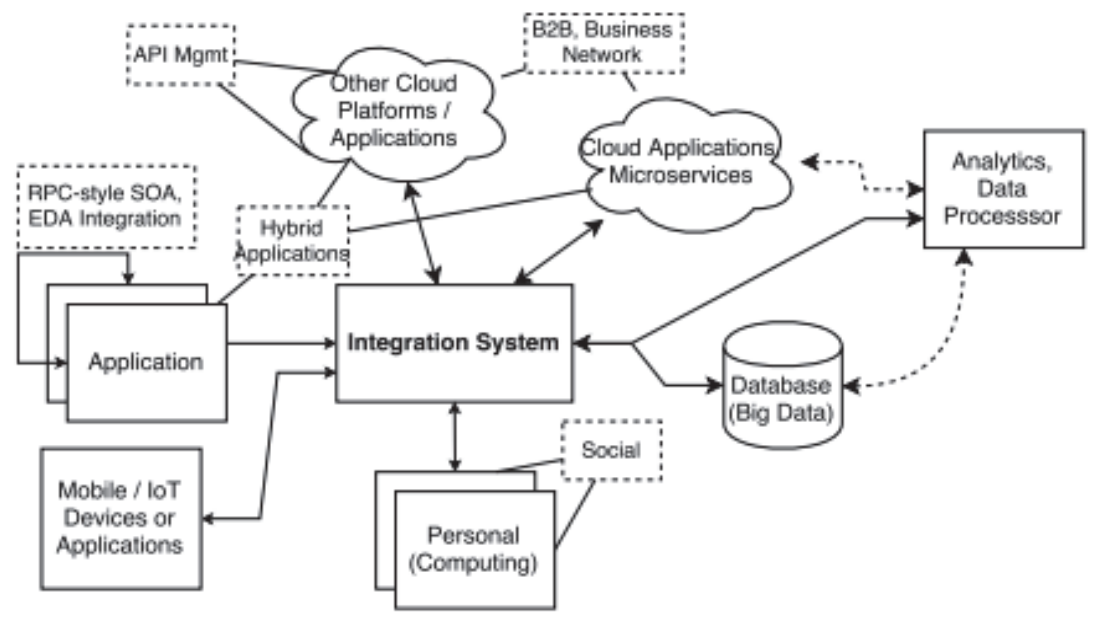

Figure 1. IT Trends since 2005 and Link with EAI

Source: (Ritter et.al, 2017)

The study findings of Fenner (2002) highlighted that there exists two chief architectures of EAI, which play an indispensable role in developing the procedure of systems integration. As identified by Fenner (2002), these two EAI architectures comprised direct point-to-point (PTP) and Middleware-based architectures. The PTP architecture of EAI is in this particular context ca $n$ be perceived as a basic and traditional approach, which is applicable to the situations, wherein few systems are required to be incorporated (Fenner, 2002). The later phases of direct point-to-point (PTP) EAI architecture can be understood from the below illustrated diagram (Figure 2).

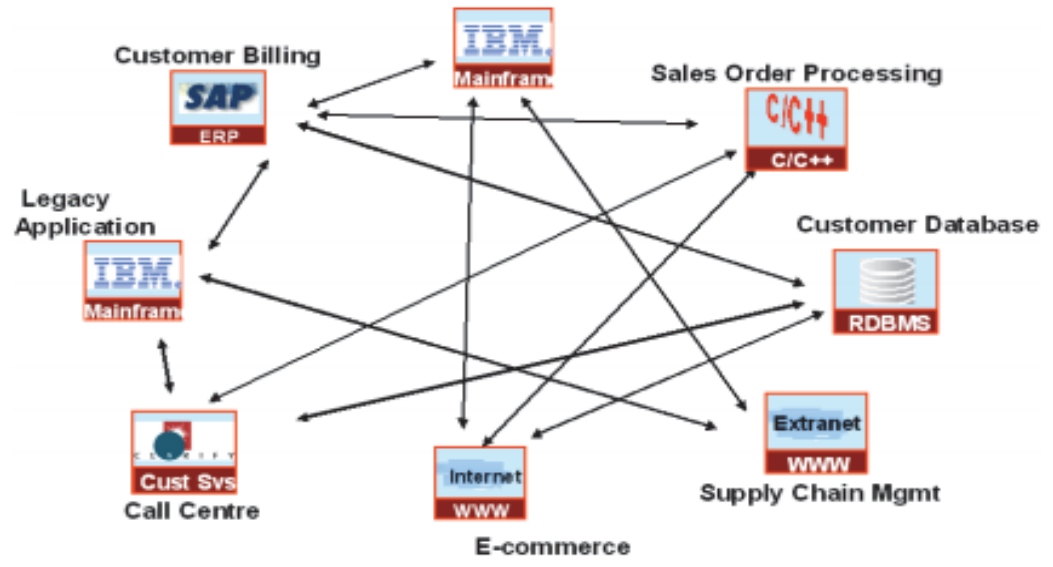

Figure 2. Later Phases of Direct point-to-point (PTP) Architecture

Source: (Fenner, 2002)

Correspondingly, in relation to the study findings presented by Fenner (2002), Middleware-based EAI infrastructure supports in the development of generic interfaces. This, in turn, facilitates the applications to pass messages to one another. The Figure 3 below focuses on representing the Middleware-based integration 
architecture of EAI:

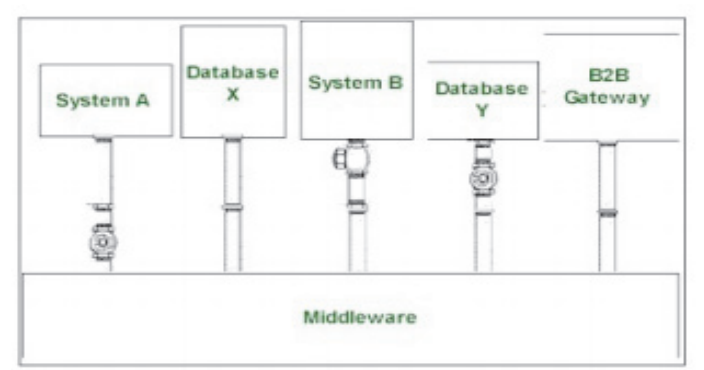

Figure 3. Middleware-Based Integration Architecture

Source: (Fenner, 2002)

\subsection{A Concise Description of Middleware Technologies}

With regard to the study conducted by Kalyani (2012), middleware is used in the circumstance of High Level Architecture (HLA) and is applied in several distributed simulations. Kalyani (2012) in the study revealed that the middleware technologies are segregated based on the factors concerning recoverability and scalability. In this context, the distinct sorts of middleware technologies are identified as Remote Procedure Calls (RPCs), SQLoriented Data Access, Application Servers, MOM, Object Request Broker (ORB), and TP Monitors among others (Kalyani, 2012). Based on the study findings of Chen et.al. (2006), RPCs help in distributing the application logics successfully throughout the available networks. On the other hand, Application Servers are viewed as the software installed on a computer for ensuring smooth running of different applications. In contrast, MOM facilitates program-to-program data exchange and sending messages to the clients as per their requirements. This particular technology of middleware is most appropriate for the applications that are event-driven in nature (Kalyani, 2012). As discussed in the study conducted by Chen et.al. (2006), ORB and TP Monitors perform the tasks of sending objects, as well as, requesting services within an object-oriented mechanism and developing distributed applications respectively. In addition, SQL-oriented Data Access technology forms, develops, and maintains a better correlation among the applications and database servers (Kalyani, 2012).

\subsection{A Short Explanation of Cloud Computing Frameworks}

Ukil et.al. (2013) stated that Cloud service is prone to facing software and statistical attacks because of its dispersed nature. Therefore, the security issue is witnessed to prevail within cloud computing at an extensive level (Ukil et.al, 2013). Subsequently, based on this notion, an integrity-ensured trusted cloud computing framework (Figure 4) has been developed by Ukil et.al. (2013) in order to address the security issue successfully.

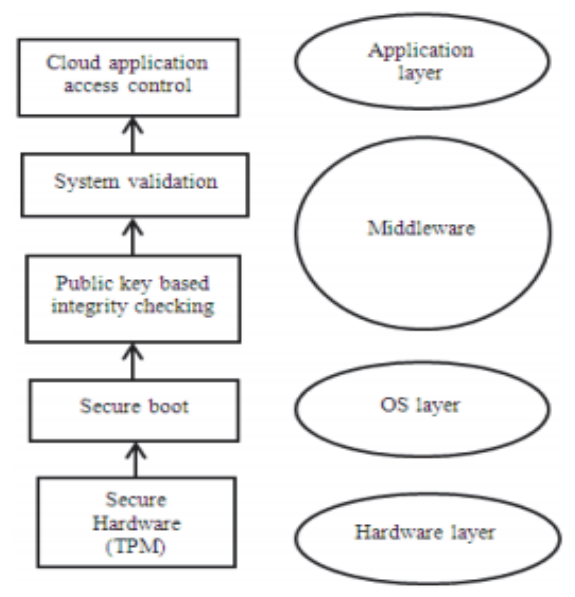

Figure 4. Integrity-Ensured Trusted Cloud Computing Framework

Source: (Ukil et al., 2013)

On the other hand, as portrayed in the study carried out by Youssef \& Alageel (2012), a rigid framework of secure cloud computing was developed for combating against any sort of attack or threats, in accordance to adhering 
privacy requirements as well. According to this study of Youssef \& Alageel (2012), the framework comprised three indispensable security elements, which includes Concerns \& Risks, Security \& Privacy Requirements, and Attacks $\&$ Threats along with their respective characteristics. This framework of secure cloud computing framework can be depicted from the Figure 5 below.

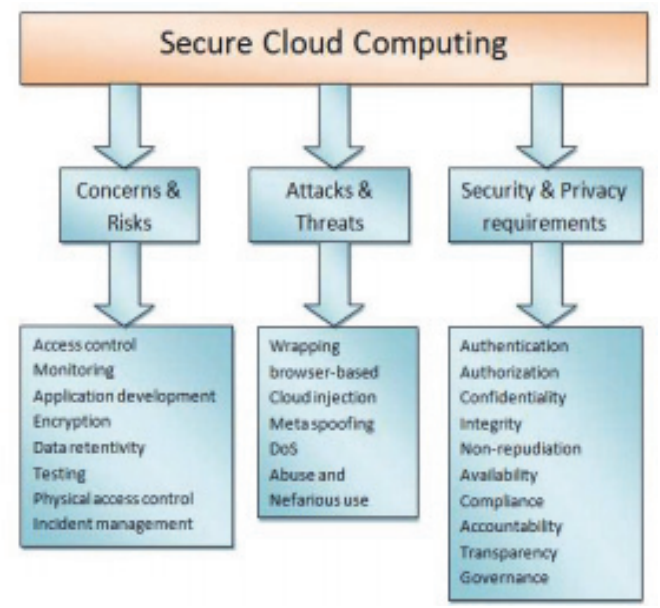

Figure 5. Secure Cloud Computing Framework

Source: (Youssef \& Alageel, 2012)

Similar to the framework of secure cloud computing, an organisational control framework can also be related to cloud computing and EAI. According to Srivastava \& Kumar (2015), this particular framework includes a Threat Index (TI), which computes the susceptibility of a cloud setting based data centre with respect to attacks and threats. A diagram (Figure 6) is presented hereunder to understand the mentioned organisational control framework in a better way.

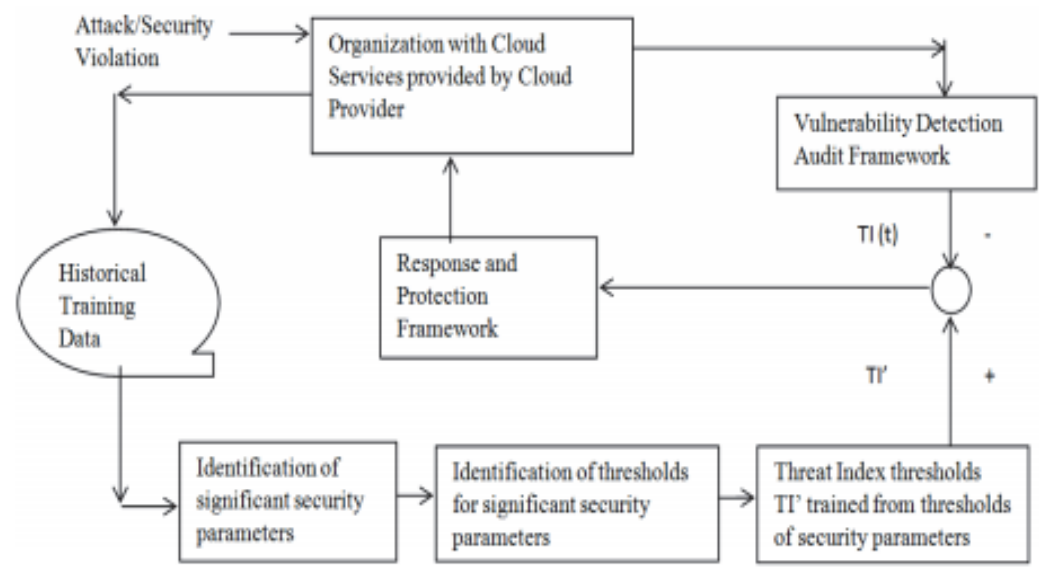

Figure 6. Organisational Control Framework

Source: (Srivastava \& Kumar, 2015)

\section{Research Method \& Data Analysis}

A qualitative research method has been adopted for this particular research paper in order to gain insights about the utilisation of different middleware technologies for cloud computing frameworks in alignment with successful EAI. This specific research method is adopted because it helps in accessing valuable data to use and address the study issue identified by exploring several significant phenomena (Blessing \& Forister, 2009). This phenomena related to the research paper fundamentally included EAI along with different types of relevant architectures, middleware technologies, and frameworks of cloud computing. It is believed that use of qualitative research method for this research paper has supported in drawing valid inferences by exploring realities and making 
pertinent observations as well (Rubin \& Babbie, 2010). Therefore, on the basis of these notions, utmost focus is levied on retrieving relevant data from different secondary sources including scholarly journals, books, articles, and authentic online reports, as well as, documents. Proper selection of literatures, in addition to the use of appropriate keywords related to the identified study issue eventually helped in maintaining validity along with reliability of the research paper.

Moreover, Data analysis for this particular research paper has been performed with the assistance of case study method. This specific method of data analysis i.e. case study proved to be appropriate, as it facilitated in exploring and understanding the complex phenomena of EAI, middleware technologies, and frameworks of cloud computing (Zainal, 2007). The case study has been finally based on two leading business corporations i.e. IBM and Microsoft, which have been using middleware technologies for developing the structures of their respective cloud computing platforms.

\section{Discussion}

\subsection{A General Idea about the Use of Middleware Technologies for Cloud Computing Frameworks}

In general, there are numerous middleware technologies that are used specifically for cloud computing frameworks. These technologies comprise Remote Data Access (RDA), Object Request Brokers (ORBs), Remote Procedure Calls (RPCs), and Distributed Transaction Processing (DTP). RDA is utilised for the frameworks of cloud computing by taking into concern a RDA protocol, which sends language statements of data manipulation to a suitable database server. This protocol and RDA both helps in enhancing the overall processing along with transporting the consequence back to the invoking procedure. On the other hand, it is evident that a standard implementation of ORB technology is identified as CORBA. ORB along with CORBA increases the probability of invoking a remote object by permitting a source item to send messages to this remote object. Another type of middleware technology i.e. RPCs is observed to be utilised in most of the services of network operating mechanism. Finally, the use of DTP technology for cloud computing frameworks can be determined from implementing execution semantics for the purpose of establishing, developing, and maintaining interactions among the end users and the servers (Khosrow-Pour, 2002).

Based on the findings obtained from the literature review relating to this particular research paper, Application Servers, TP Monitors, SQL-oriented Data Access, and MOM are the other technologies of middleware, which can be used for the frameworks of cloud computing (Kalyani, 2012; Chen et.al., 2006). Utilisation of these identified middleware technologies for cloud computing frameworks is usually made possible by creating an association amid applications and database servers. In addition, it can also be used by developing program logics based on remote systems and by ensuring successful exchange of messages between the software programs. It is expected that proper and accurate utilisation of these middleware technologies helps in supporting the clients to access various web servers and, thereby, adapt to the constantly transforming business setting (Kalyani, 2012). The below Figure 7 reflects the concept of using middleware technologies.

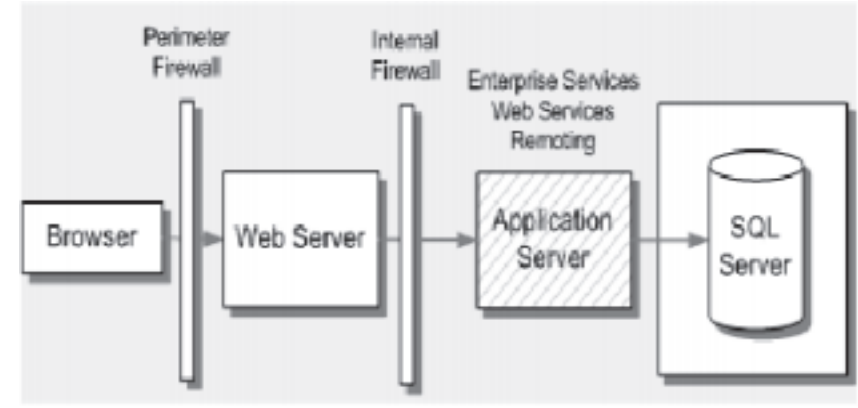

Figure 7. Expansion of EAI and Middleware Technologies

Source: (Kalyani, 2012)

\subsection{Use of Middleware Technologies by IBM for Cloud Computing Frameworks}

International Business Machines Corporation, popularly acknowledged as IBM, is an American based global technology business firm. The company performs its operations through some specific business segments that embrace cloud platforms, worldwide financing, and hardware, as well as, software solutions among others (Reuters, 2018). Application Programming Interface (API) is one of the middleware technologies, which is used by IBM in 
the cloud computing from the perspective of EAI. This particular technology allows the developers of the company to control, publish, subscribe to, secure, and design APIs to the maximum possible extent. IBM API Management, also signified as APIM or IBM APIM, facilitates the modern business corporations to circulate their business services particularly to the external, as well as, internal application developers throughout the public and private cloud settings (Bernard, 2018).

Successful execution of IBM API Management covers certain other major operational areas as well. This includes lifecycle administration, gateway integration of API for runtime enforcement, usage analytics, and developer portal. It is claimed that with the help of using APIM, it is the application developers who can easily browse an incorporated API portal and reap certain other benefits as well such as registering applications in an efficient way. Moreover, the other benefits encompass invoking APIs, examining their individual applications, participating in an incorporated discussion forum and rating along with making comments on the use of APIs (Bernard, 2018).

Apart from APIM, IBM also explores DevOps as the other middleware technological solution, which plays a major part in fostering closer alliance amid the respective business lines, development, and IT operations perform by the company globally. Use of DevOps, which is a type of middleware technology, certainly contributes in the development of agility and reducing the time required to address the feedbacks of the end users. It is believed that with continuous deployment, delivery, and monitoring of DevOps, IBM can make faster response to the respective business markets and, thereby, gain positive user experiences. By scaling DevOps efficiently without affecting the business, it might form, develop, and preserve start-up culture. Additionally, it can also help in binding business, advancement, and operations together in the procedure of accomplishing desired organisational targets within a definite timeframe (IBM, 2018).

Another middleware technology, which is applied by IBM for cloud computing frameworks, is the IBM WebSphere ${ }^{\circledR}$ Application Server. It is considered to be the foremost open standards-based application foundation, which takes pioneering applications, unrivalled operational competence, security, management, control, and reliability into considerations. With the support of using IBM WebSphere ${ }^{\circledR}$ Application Server, it is argued that the modern organisations can form, as well as, combine the applications of a business-critical enterprise with original new functionalities (IBM, 2017).

\subsection{Utilisation of Middleware Technologies by Microsoft for Cloud Computing Frameworks}

Similar to IBM, another foremost business corporation, which uses middleware technologies for the frameworks of cloud computing is Microsoft. The company was initiated in the year 1975 and is regarded as one of the foremost providers of services, software, and solutions particularly to the customers, as well as, the businesses at a global level (EMC Corporation, 2013). Using of the middleware technologies is not a new concept for Microsoft, as the company desired to make these technologies quite different from others for the purpose of retaining competitiveness. During the year 2002, .NET Remoting became the most widely used middleware in Microsoft, which played an important role in connecting people, mechanisms, and services through web with the assistance of tools and libraries (Etzkorn, 2017).

Moreover, the company launched another middleware technology i.e. Windows Communication Foundation in 2006, which helped Microsoft to attain superior level of competitive position in the respective industry. The technology business corporation introduced a virtual machine named the Microsoft Common Language Runtime (CLR), which is executed by all .NET routines. This middleware technology employs a setting of program code execution, which is fundamentally described by the notion of Common Language Infrastructure (CLI). The CLR technology is typically based on Microsoft and .NET, which is mostly utilised for incorporating dissimilar programming languages into the systems by stating certain rules for these languages to follow and thereby ensuring greater interaction amid the same (Etzkorn, 2017).

It is evident that Microsoft uses Microsoft Application Platform as a middleware technology in order to develop operational services along with creating higher extent of business and customer value. This particular technological platform is regarded as a wide-ranging and incorporated set of technologies and tools, which focuses on maximising the value of application portfolio of the company. Application platforms of Microsoft contain three specific layers such as infrastructure, database, and middle tier (Microsoft Corporation, 2017). This has been described hereunder in the form of a diagram. 


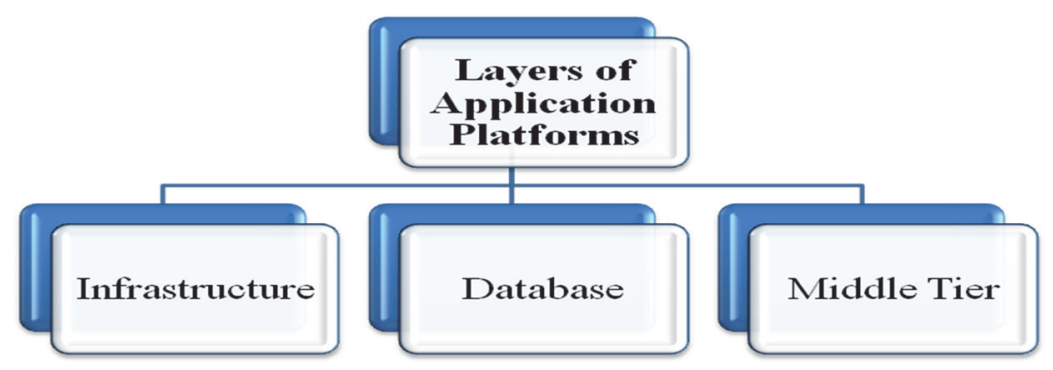

Figure 8. Three layers of application platforms

Source: (Microsoft Corporation, 2017)

Based on the above portrayed diagram (Figure 8), the infrastructure layer forms the basis of an application platform, which offers virtualisation, networking services, access, core security, and identity. On the other hand, the database layer takes into concern infrastructure services for the purposes of storing, retrieving, and examining application data. Database systems perform various significant functions, which fundamentally comprise gathering data from manifold systems, processing application transactions, and developing tools for ensuring successful analysis, as well as, reporting. Finally, the middle tier layer delivers business logic and establishes connections among the operational systems and applications. It is in this particular layer that the application servers handle transactions successfully, which occur between systems and applications (Microsoft Corporation, 2017).

\section{Conclusion}

Based on the findings retrieved from literature review and secondary sources, it has been evident that the modern business corporations are more inclined towards using middleware technologies for cloud computing frameworks from the perspective of EAI. These types of technologies are mostly applied to manifold distributed simulations, which are often observed to play an essential role for developing the available networks. The distinct middleware technologies, which are used by the business entities in the present day contexts, are RPCs, Application Servers, TP Monitors, and MOM among others. There lays the presence of numerous cloud computing frameworks that eventually justify the application of middleware technologies in the respective operations. These frameworks comprised integrity-ensured trusted cloud computing framework and secure cloud computing framework.

With regard to the findings obtained from case study analysis method in this particular research, it is evident that two renowned technology based companies namely IBM and Microsoft are indulged in applying middleware technologies within their respective cloud settings. For example, IBM uses the middleware technologies of APIM and DevOps, whereas, Microsoft utilises .NET Remoting and CLR as effective technologies related to middleware. The findings obtained for this research are deemed to be reliable due to its authenticity. Consideration of collecting primary data either via survey or interview or both may raise the future scope of this research.

\section{References}

Bernard, A. (2018). Exploring IBM API Management, Middleware for App Integration. Feature. Retrieved February 4, 2018, from http://searchmicroservices.techtarget.com/feature/Exploring-IBM-API-Managementmiddleware-for-app-integration

Blessing, J. D., \& Forister, J. G. (2009). Introduction to Research and Medical Literature for Health Professionals. Jones \& Bartlett Publishers.

Chen, Q., et al. (2006). Middleware Components for E-commerce Infrastructure: An Analytical Review. Issues in Informing Science and Information Technology, 3, 137-145.

Chen, R. (2010). Intelligent Computing and Information Science: International Conference, ICICIS 2011, Chongqing, China, January 8-9, 2011. Proceedings, Part 1. Springer Science \& Business Media.

EMC Corporation. (2013). Overview. Microsoft Corporation. Retrieved February 5, 2018, from https://www.emc.com/collateral/customer-profiles/h11612-cp-microsoft-corporation.pdf

Etzkorn, L. H. (2017). Introduction to Middleware: Web Services, Object Components, and Cloud Computing. CRC Press.

Fenner, J. (2002). EAI Integration Architectures. Enterprise Application Integration Techniques. Retrieved February 4, 2018, from http://www0.cs.ucl.ac.uk/staff/ucacwxe/lectures/3C05-02-03/aswe21-essay.pdf 
IBM. (2017). WebSphere Application Server for IBM i. Software. Retrieved February 4, 2018, from https://www03.ibm.com/systems/power/software/i/websphere/

IBM. (2018). What is DevOps? Cloud. Retrieved February 4, 2018, from https://www.ibm.com/cloud/devops

Kalyani, K. (2012). Recent Trends and Challenges in Enterprise Application Integration. International Journal of Application or Innovation in Engineering \& Management (IJAIEM), 1(4), 62-70.

Khosrow-Pour, M. (2002). Issues \& Trends of Information Technology Management in Contemporary Organizations. Idea Group Inc (IGI).

Microsoft Corporation. (2017). Microsoft Application Platform. Platform Modernization Alliance. Retrieved February 4, 2018, from https://www.platformmodernization.org/Pages/platform.aspx

MuleSoft, Inc. (2018). Understanding Enterprise Application Integration - The Benefits of ESB for EAI. Enterprise Integration. Retrieved February 4, 2018, from https://www.mulesoft.com/resources/esb/enterpriseapplication-integration-eai-and-esb

Nardi, J. C. et al. (2011). Foundational Ontologies for Semantic Integration in EAI: A Systematic Literature Review. Springer-Verlag.

Reuters (2018). Full Description. Profile: International Business Machines Corp (IBM). Retrieved February 4, 2018, from https://www.reuters.com/finance/stocks/company-profile/IBM

Ritter, D., et al. (2017). Introduction. Patterns for Emerging Application Integration Scenarios: A Survey, pp. 1-5.

Rubin, A., \& Babbie, E. R. (2010). Research Methods for Social Work. Cengage Learning.

Srivastava, H. \& Kumar, S. A. (2015). Control Framework for Secure Cloud Computing. Journal of Information Security, 6, 12-23.

Ukil, A., et al. (2013). A Security Framework in Cloud Computing Infrastructure. International Journal of Network Security \& Its Applications (IJNSA), 5(5), 11-18.

Van den Bosch, et al. (2010). A Selection-Method for Enterprise Application Integration Solutions. Springer-Verlag.

van Sinderen, et al. (2012). Enterprise Interoperability: 4th International IFIP Working Conference, IWEI 2012, Harbin, China, September 6-7, 2012, Proceedings. Springer.

Youssef, A. E., \& Alageel, M. (2012). A Framework for Secure Cloud Computing. International Journal of Computer Science Issues, 9(4), 490-496.

Zainal, Z. (2007). Case Study as a Research Method. Journal Kemanusiaan Bil, 9, 1-2.

\section{Copyrights}

Copyright for this article is retained by the author(s), with first publication rights granted to the journal.

This is an open-access article distributed under the terms and conditions of the Creative Commons Attribution license (http://creativecommons.org/licenses/by/4.0/). 